\title{
An Index Theorem for Super Derivations *
}

\author{
Arthur Jaffe and Andrzej Lesniewski \\ Harvard University, Cambridge, MA 02138, USA
}

\section{Dedicated to Roland Dobrushin}

\begin{abstract}
We show that the Chern character given by a super-KMS functional on a quantum algebra can be interpreted in terms of the index of a super derivation on a projection of the algebra.
\end{abstract}

\section{Introduction}

The heat kernel representation of the index of a Fredholm operator provides a natural connection between statistical physics (through the partition function) and geometry (regarding the index as a topological invariant). The equivalence in certain statistical mechanics models between the Gibbs variational principles (as expressed through the equation of Dobrushin, Lanford and Ruelle) and the KMS condition provided a fundamental interpretation of trace invariants for operator algebras. Recently the importance of the KMS property (in a super or graded setting) has emerged as a fundamental starting point for the definition of a class of geometric invariants. This subject unifies Connes' noncommutative differential geometry, analysis in an infinite dimensional setting, and ideas from statistical and particle physics. Thus it is especially appropriate to dedicate this note to Roland Dobrushin.

Our purpose here is to investigate an aspect of index theory for a super derivation without assuming that it is generated by a Fredholm operator, without assuming compactness or a bound on the dimension of an underlying manifold, and without necessarily obtaining integral invariants. In place of the standard assumptions we suppose that we are given a super-KMS functional on a quantum algebra $\mathscr{A}$ (defined below). This elementary assumption leads to a Chern character $\tau$ for the quantum algebra, and a pairing between $\tau$ and the $K_{0}$ group of the even part of the algebra. We show here that this pairing can be interpreted in terms of the

* Supported in part by the National Science Foundation under Grant DMS/PHY 8816214 
index theory for a super derivation on a projection of the quantum algebra. This is a generalization of a related result when the square of the super derivation is inner and arises from a trace-class heat kernel [C2]. This yields an interpretation of the pairing between $K_{0}\left(\mathscr{A}_{+}\right)$and $\tau$ in the super-KMS setting, related of course to Connes' theory for entire cyclic cohomology.

\section{The Projection of a Quantum Algebra}

Let $(\mathscr{A}, \Gamma, \alpha, d)$ be a quantum algebra. Thus $\mathscr{A}$ is a unital $C^{*}$-algebra with a $\mathbb{Z}_{2}$ grading $\Gamma$. Also $\alpha_{t}$ is a continuous, one-parameter automorphism group of $\mathscr{A}$, which commutes with $\Gamma$. The infinitesimal generator of $\alpha_{t}$ is $d^{2}=-\left.i \frac{d}{d t} \alpha_{t}\right|_{t=0}$, where $d$ is a super derivation on $\mathscr{A}$. Let $e$ be an even, self-adjoint, idempotent in the domain of $d$,

$$
e^{2}=e=e^{*}=e^{\Gamma} \in \mathscr{D}(d) \subset \mathscr{A} .
$$

Define $\mathscr{A}^{e}$ as the unital $C^{*}$ algebra $e \mathscr{A} e$ with the unit element $e$.

Below we establish the connection between the Chern character $\tau$ defined by a super-KMS functional on the quantum algebra $(\mathscr{A}, \Gamma, \alpha, d)$ and the index of a super derivation $d^{e}$ which we define on $\mathscr{A}^{e}$.

We define $d^{e}$ on the domain $\mathscr{D}(d) \cap \mathscr{A}^{e}$ by

$$
d^{e} a=e(d a) e .
$$

Lemma 1. $d^{e}$ is a super derivation on $\mathscr{A}^{e}$ and $d^{e} e=0$.

Proof. A super derivation has two properties: it anticommutes with the grading $\Gamma$ and $d^{e}(a b)=\left(d^{e} a\right) b+a^{\Gamma}\left(d^{e} b\right)$. The first property is a consequence of (1) and the fact that $d$ is a super derivation. The graded Leibniz rule follows from these properties as well. To establish $d^{e} e=0$, we remark that $e^{2}=e$ ensures

$$
(d e) e+e d e=d e .
$$

Multiplying on the right by $e$ we obtain

as claimed.

$$
d^{e} e=e(d e) e=0,
$$

Let us define an element $q, q \in \mathscr{A}_{-}$, the odd part of $\mathscr{A}$, by the equation

$$
q=e d e-(d e) e .
$$

Define a super derivation $\delta_{q}$ on $\mathscr{A}$ by

$$
\delta_{q}(a)=q a-a^{\Gamma} q
$$

which yields a bounded perturbation $d_{q}$ of $d$ given by

$$
d_{q}=d+\delta_{q} .
$$

In [JLW] we established that $D_{q}=\left(d_{q}\right)^{2}$ is the infinitesimal generator of a contiuous, even, one parameter automorphism group $\alpha_{t}^{q}$ of $\mathscr{A}$. Here $\alpha_{t}^{q}=\exp \left(\right.$ it $\left.\operatorname{ad}\left(D_{q}\right)\right)$, and $\alpha_{t}^{q}$ commutes with $\Gamma$. 
Proposition 2. The group $\alpha_{t}^{q}$ acts on $\mathscr{A}^{e}$ and $\alpha_{t}^{q}(e)=e$. Furthermore, $d_{q} e=0$.

Proof. Using (4), we note that

$$
d_{q} e=d e+q e-e q=d e-(d e) e-e d e .
$$

By (3), we have $d_{q} e=0$. It follows that $\alpha_{t}^{q}(e)=e$. For $a=e a e$, we have

$$
\alpha_{t}^{q}(a)=\alpha_{t}^{q}(e a e)=e \alpha_{t}^{q}(a) e,
$$

so $\alpha_{t}^{q}$ maps $e \mathscr{A} e$ into itself.

Proposition 3. On $\mathscr{A}^{e} \cap D(d)$,

$$
d_{q}=d^{e} .
$$

Proof. For $a=e a e \in \mathscr{D}(d)$ we have by Proposition 2,

$$
d_{q} a=d_{q}(e a e)=e\left(d_{q} a\right) e .
$$

But $e q e=e(e d e-(d e) e) e=0$. Thus

$$
e q a e=e q e a e=0,
$$

and likewise eaqe $=0$. Hence

$$
d_{q} a=e(d a+q a-a q) e=e(d a) e=d^{e} a,
$$

as claimed. Thus we have

Corollary 4. The derivation $D_{e}=\left(d^{e}\right)^{2}$ on $\mathscr{A}^{e}$ can be exponentiated to the continuous automorphism group

$$
\alpha_{t}^{e}=\exp \left(\text { it } D_{e}\right)=\exp \left(i t D_{q}\right) \uparrow \mathscr{A}^{e} .
$$

Furthermore, we now have verified

Theorem 5. $\left(\mathscr{A}^{e}, \Gamma, \alpha_{t}^{e}, d^{e}\right)$ is a quantum algebra.

\section{The Projection of a Super-KMS Functional}

Let $\omega$ be a super-KMS functional on the quantum algebra $\left(\mathscr{A}, \Gamma, \alpha_{t}, d\right)$. In other words, $\omega$ is a continuous linear functional on $\mathscr{A}$ such that on the subalgebra $\mathscr{A}_{\alpha}$ of entire elements for $t \rightarrow \alpha_{t}$,

$$
\omega(d a)=0 \quad \text { and } \quad \omega(a b)=\omega\left(b^{\Gamma} \alpha_{i}(a)\right) .
$$

Let $\omega_{e}$ be a functional on $\mathscr{A}^{e}$ defined by

$$
\omega_{e}(a)=\omega^{q}(a)
$$

where $\omega^{q}$ is the super-KMS functional for $\left(\mathscr{A}, \Gamma, \alpha_{t}^{q}, d_{q}\right)$ constructed in [JLW] by perturbation theory and analytic continuation of the wave operator.

Theorem 6. $\omega_{e}$ is a super-KMS functional for $\left(\mathscr{A}^{e}, \Gamma, \alpha_{t}^{e}, d^{e}\right)$. 
Proof. Clearly $\omega_{e}$ is continuous on $\mathscr{A}^{e}$. Thus we need only check the analog of (10). Clearly

$$
\omega_{e}\left(d^{e} a\right)=\omega^{q}\left(e d^{e} a e\right)=\omega^{q}\left(e d_{q} a e\right)=\omega^{q}\left(d_{q}(e a e)\right)=0
$$

Also

$$
\begin{aligned}
\omega_{e}(a b) & =\omega^{q}(e a b e)=\omega^{q}\left(e a e^{2} b e\right)=\omega^{q}\left(e b^{\Gamma} e \alpha_{i}^{q}(a) e\right) \\
& =\omega_{e}\left(b^{\Gamma} \alpha_{i}^{q}(a)\right)=\omega_{e}\left(b^{\Gamma} \alpha_{i}^{e}(a)\right) .
\end{aligned}
$$

In the last step we use Corollary 4 to replace $\alpha_{t}^{q}$ by $\alpha_{t}^{e}$, and we then analytically continue $\omega_{e}\left(b^{\Gamma} \alpha_{t}^{q}(a)\right)=\omega_{e}\left(b^{\Gamma} \alpha_{t}^{e}(a)\right)$ to the point $t=i$.

\section{The Index of a Super Derivation}

The index of the super derivation $d^{e}$ on a quantum algebra $\left(\mathscr{A}^{e}, \Gamma, \alpha^{e}, d^{e}\right)$ is defined with respect to a super-KMS functional $\omega_{e}$ by

$$
i_{\omega_{e}}\left(d^{e}\right)=\omega_{e}(\mathbf{1})=\omega_{e}(e) .
$$

The existence of the super-KMS property in part replaces a "Fredholm condition;" it ensures the existence of (12), although the index defined by (12) may not be integral.

An even cochain $\left\{f_{n}\right\}$ is cyclic if for each $n$,

$$
f_{n}\left(a_{0}, \ldots, a_{n}\right)=(-1)^{n} f_{n}\left(a_{n}^{\Gamma}, a_{0}, \ldots, a_{n-1}\right) .
$$

Furthermore, and entire cocycle $\tau$ is said to be normalized if

$$
f_{n-1}\left(a_{0}, \ldots, a_{n-1}\right)=\tau_{n}\left(\mathbf{1}, a_{0}, \ldots, a_{n-1}\right)+(-1)^{n+1} \tau_{n}\left(a_{0}, \ldots, a_{n-1}, \mathbf{1}\right)
$$

is cyclic. Connes has proved that every entire cocycle $\tau$ is cohomologous to a normalized entire cocycle $\hat{\tau}$ with the property that $\tau_{0}=\hat{\tau}_{0}$, see Lemma 6 of [C2] and its proof. If $e$ is an even projection in $\mathscr{A}$ and $\tau$ is an entire cocycle, define

$$
\langle\tau, e\rangle=\sum_{n=0}^{\infty}(-1)^{n} \frac{(2 n) !}{n !} \hat{\tau}_{2 n}(e, e, \ldots, e),
$$

where $\tau$ corresponds to $\hat{\tau}$ as above. Now let us take for $\tau$ the Chern character on $\mathscr{A}$ constructed from $\omega$ in [JLO1, K, JLO2].

Theorem 7. With the above definitions, $\langle\tau, e\rangle$ is well-defined and

$$
\langle\tau, e\rangle=i_{\omega_{e}}\left(d^{e}\right)
$$

Proof. We remark that $\langle\tau, e\rangle$ is independent of the choice of normalized, cohomologous cocycle $\hat{\tau}$. In fact, any two choices $\hat{\tau}_{1}$ and $\hat{\tau}_{2}$ differ by a coboundary $\partial G$, which is normalized. It follows from Lemma 7 of [C2] that $\langle\partial G, e\rangle=0$, showing that the pairing $\langle\tau, e\rangle$ is well defined. Similarly, for given $e,\langle\tau, e\rangle$ depends only on the cohomology class of $\tau$.

We deform $\tau$ by deforming $d$ to $d_{q}$. By [JLW], this deformation yields $\tau^{q}$ cohomologous to $\tau$. But $\tau^{q} \uparrow \mathscr{A}^{e}$ is the Chern character constructed from $\omega_{e}$, which by $d^{e} e=0$ and $\tau_{0}^{q}=\hat{\tau}_{0}^{q}=\omega_{e}$ yields (14). 
Remark. Consider the case where $\mathscr{A}$ is an algebra of operators on a Hilbert space $\mathscr{H}$, and where $d a$ is given by the graded commutator

$$
d a=[Q, a] \equiv Q a-a^{\Gamma} Q,
$$

with $Q$ an odd, self-adjoint, Fredholm operator on $\mathscr{H}$. Then for $a \in \mathscr{A}^{e}$,

$$
d^{e} a=[e Q e, a] .
$$

In this case, Connes established

$$
\langle\tau, e\rangle=i_{\omega_{e}}\left(d^{e}\right)=\operatorname{Index}\left((e Q e)_{+}\right),
$$

where Index denotes the Atiyah-Singer index and + denotes the component of $e Q e$ which maps $\frac{1}{2}(I+\Gamma) \mathscr{H}$ to $\frac{1}{2}(I-\Gamma) \mathscr{H}$.

Let $\mathscr{A}_{k}=\operatorname{Mat}_{k}(\mathscr{A})$ denote the algebra of $k \times k$ matrices with entries in $\mathscr{A}$, and let $\mathscr{A}_{k}^{e}=e \mathscr{A}_{k} e$, where $e \in\left(\mathscr{A}_{k}\right)_{+}$is a self-adjoint projection. One can perform the above construction as well on $\mathscr{A}_{k}^{e}$, as follows. The super derivation $\tilde{d}^{e}=e \tilde{d} a e$ can be defined, using $(\tilde{d} a)_{i j}=d a_{i j}$. Furthermore, a super-KMS functional $\omega$ on $(\mathscr{A}, \Gamma, \alpha, d)$ extends to $\tilde{\omega}$ on $\left(\operatorname{Mat}_{k}(\mathscr{A}), \tilde{\Gamma}, \tilde{\alpha}, \tilde{d}\right)$, where $\tilde{\Gamma}=\Gamma \otimes \mathrm{Id}, \tilde{\alpha}=\alpha \otimes \mathrm{Id}$, by

$$
\tilde{\omega}(a)=\sum_{i=1}^{n} \omega\left(a_{i i}\right) \text {. }
$$

Clearly $\tilde{\omega}$ is super-KMS, as follows from

and

$$
\tilde{\omega}(\tilde{d} a)=\sum_{i} \omega\left(\tilde{d} a_{i i}\right)=0
$$

$$
\tilde{\omega}(a b)=\sum_{j} \omega\left((a b)_{j j}\right)=\sum_{j, k} \omega\left(a_{j k} b_{k j}\right)=\sum_{j, k} \omega\left(b_{k j}^{\Gamma} \alpha_{i}\left(a_{j k}\right)\right)=\tilde{\omega}\left(b^{\Gamma} \tilde{\alpha}_{i}(a)\right) .
$$

More generally, if $f$ is an entire cyclic cochain on $\mathscr{A}$, then $\tilde{f}=f \# \operatorname{Tr}$ is an entire cyclic cochain on $\mathscr{A}_{k}$ [C2]. Note that Connes' growth condition guarantees that the series (13) defining $\langle\widetilde{f}, e\rangle$ converges. By the previous arguments, we have

Corollary 8. (General Index Formula).

$$
\langle\tilde{\tau}, e\rangle=\tilde{\omega}_{2}\left(\tilde{d}^{e}\right) .
$$

This formula is our general index theorem representing the value of $\tau$ evaluated on an element of $K_{0}\left(\mathscr{A}_{+}\right)$as the index of a super derivation.

\section{References}

[C1] Connes, A.: Noncommutative differential geometry. Publ. Math. IHES 62, 257-360 (1985)

[C2] Connes, A.: Entire cyclic cohomology of banach algebras and characters of $\theta$-summable Fredholm modules. K-Theory 1, 519-548 (1988)

[JLO1] Jaffe, A., Lesniewski, A., Osterwalder, K.: Quantum $K$-theory. I. The Chern character. Commun. Math. Phys. 18, 1-14 (1988)

[JLO2] Jaffe, A., Lesniewski, A., Osterwalder, K.: On super-KMS functionals and entire cyclic cohomology. K-Theory (to appear) 
[JLW] Jaffe, A., Lesniewski, A., Wisniowski, M.: Deformation of super-KMS functionals. Commun. Math. Phys. 121, 527-540 (1989)

[K] Kastler, D.: Cyclic cocyles from graded KMS functionals. Commun. Math. Phys. 121, 345-350 (1989)

Communicated by A. Jaffe

Received March 20, 1989; in revised form April 11, 1989 